\title{
Impact Velocity Prediction in a Traffic Accident
}

\author{
Ali Can Yilmaz and Kadir Aydin \\ Cukurova University, Automotive Engineering Department, 01330 Adana, Turkey
}

\begin{abstract}
Reconstruction of traffic accidents has been so crucial scientific process in order to make impartial and judicious decisions. This study focuses on impact speed prediction of accident sufferers just before the collision in a comprehensive scientific way by using an accident reconstruction software called "vCrash" and Function Fitting Neural Network (FITNET) artificial intelligence method (predictor) in case of absence of skid marks or other clues about calculating impact speeds. A sample real world accident was simulated on the software several times by changing collision speeds to form different deformation on the collision regions of the vehicles in every simulation. Every single deformation amount corresponding to each impact velocity was recorded and used as teaching data for FITNET prediction model. Using 10-fold cross validation, mean squared error (MSE) and multiple correlation coefficients $(\mathrm{R})$ were observed to exhibit performance of the prediction model. The model performed high $\mathrm{R}$ (close to 1) and acceptable MSE values. This method aims that, in a probable similar accident scene in future, it will be possible to analyze the impact speeds just by entering average deformation amounts into an application on a portable device at the accident scene without requirement of expensive reconstruction tools and it will be a guide for analysis of other accident types.
\end{abstract}

\section{Introduction}

Traffic engineering focuses on safety and efficiency in transportation. Public agencies strive to reduce traffic accidents on roadways in most countries all over the world. Financial burden of traffic accidents places another problem on society. Driver and roadway design are the major factors in a traffic accident. Safety strongly depends on the gender and the age of the driver [1]. Death rates related to diseases are prone to fall in developed countries in which deaths due to traffic accidents represent a notable exception. As a country flourishes, growing economy brings a substantial increment in motor vehicles which causes substantial probable accidents. Traffic accidents are the first cause of injury-related deaths all around the world. April 7 was devoted to prevention of traffic injuries by World Health Organization (WHO) in 2004. As in many countries, in Turkey, fatalities and injuries due to traffic accidents are major problems for public [2]. Reckless driving with excessive speed gives rise to most of the traffic accidents [3]. According to WHO, traffic fatalities will be the sixth leading cause of death worldwide by the year 2020 [4].

According to 2002 annual reports, traffic accidents are the 11th reason for fatalities in Turkey. Traffic accidents have the 2 nd priority for fatalities at the age interval of 5-29 and the 3rd for the age interval of 30-44. In 1999, while the number of registered traffic accidents was 466000, it was 501000 in year 2000. General Directorate of Highway reports indicate 2954 fatalities and 94497 injuries involving 409407 accidents in year 2001 and 570419 accidents in year 2005 [5].

Transport policy substantially corresponds to accessibility, safety and environmental issues. Safety policies are of great importance almost for all countries worldwide [6]. There are some methods in evaluating plans based on infrastructure and Cost-Benefit Analysis (CBA) is now deemed the standard method in most countries [7].

Almost all of the passenger and freight transportation are carried out by highway in Turkey. In Western countries, this load on highways is shifted to railway and seaway, thus lowered highway accidents can be provided. In Turkey, highway transportation constitutes the biggest place within transportation types which unfortunately results increased number of highway vehicles [8].

Rapid increment in motor vehicles is inevitable especially during the last decades which leads to increased traffic density, several traffic accidents. Therefore, accident reconstruction is crucial in terms of solving the question of liability for these accidents. Accident experts investigate accident scenes and generally conclude about causes and procession of the accident referring to police reports. However, growing vehicle numbers and accidents make challenges for police officers to report the scene accurately. Furthermore, anti-lock braking system (ABS) in modern vehicles, when it is active, avoids locking wheels by controlling wheel slip which entails missing brake traces left on the road surface. However, brake traces are one of the most 
important clues for accident investigators to reconstruct the vehicle motion leading to an accident. More complex situations occur for vehicles equipped with vehicle dynamic control (VDC). Moreover, there are systems interfering the latest systems into the steering system to manipulate the steering system automatically [9]. There are many uncertain factors in road traffic accidents. It is necessary to consider the uncertainty influence of these factors by random theory to improve the analysis veracity. In order to improve the precision and reliability of traffic accident analysis and reconstruction, random theory and the probabilistic perturbation method are introduced for the uncertainty analysis of traffic accident. Precision and uncertainty analyses of accidents yield expedient results within expected confidence levels which also aid accident reconstruction [9].

There have been many studies about traffic accident analysis and reconstruction. Kallberg [10] conducted an analysis in Finland and made a report related to his investigations. The report is mainly for the use of the Finnish road accident investigation teams. Formulas for calculating pre-crash speeds from skid marks are presented as well as other methods for estimating speeds and locations of vehicles during different phases of the accident process. Instructions for graphic description of the pre-crash movements of vehicles are also presented. A method for determining the causes of the accident on the basis of accident reconstruction is suggested. Buck et al. [11] showed the benefits of the 3D documentation and computer-assisted, drawn-to-scale 3D comparisons of the relevant injuries with the damages to the vehicle in the analysis of the course of accidents, especially with regard to the impact situation on two examined cases. Davis also [12] studied on attempts to explain the probable reasons of accidents with physical properties and Bayesian networks statistical models together with the aid of artificial intelligence. $\mathrm{Xu}$ et al. [13] studied on effects of vehicle impact speed in pedestrian-vehicle accident by comprising reconstruction model and they verified their analysis by ten real-world accident cases to validate their results and to yield an approach for investigators. Raedt and Ponjaert [14] made investigations on predicting atfault car accidents of older drivers and they concluded that although accident prediction is difficult, the predictability of car accidents by neurocognitive measurements and a road test increases when the kind of accident is specified. Yannis et.al. conducted [15] an analysis on driver age and vehicle engine size effects on fault and severity in young motorcyclists accidents. They concluded that accident severity modeling revealed a significant second-order interaction between severity, driver age and two-wheeler engine size. On the contrary, no second-order effects were identified in fault risk modeling. Moreover, a significant effect of driver age on accident fault risk was identified. The effect of engine size was not significant.

\section{Examinations and analysis}

\subsection{Analysis of a sample accident}

A passenger car (Aston Martin DB9) and a bus (Volvo $\mathrm{B} 10 \mathrm{~B})$ were crashed into each other on an intersection within simulation software. A sample snapshot and instant data related to vehicles are shown in Fig. 1 and Fig. 2 (specifications that are not legible in the figure are depicted under figure captions in italic).

In case of absence of skid marks (mostly anti-lock braking system equipped vehicles), the biggest clue about the speeds was the damage formed on the vehicles. More damage on the vehicle(s), more energy transformed into deformation energy which is defined as crush depth $(\varepsilon)$ in terms of meters. For the scenario, vehicles were crashed into each other at angle of $90^{\circ}$ with estimated initial speeds and deformations were recorded. Coefficient of friction $(\mu)$ on the road surface was assumed as 1 . Vehicles with features similar to those in real-world accident were exposed to different deformations (minimum of 500 data for each scenario) that also corresponded to varied speed values. While one of speeds was kept constant, the other's was increased by $5 \mathrm{~km} / \mathrm{h}$. Currently in Turkey, determination of impact velocities is conducted by examining skid marks "if applicable". Considering most of the vehicles are equipped with antilock braking system, skid marks may not form on the road surface. In this case, speed analysis cannot be achieved and the situation avoids having contact with scientific analysis. Thus, in simulation, for every speed value, the simulation was repeated and the crush depths were recorded from the simulation report. One of the speed values was kept constant and the other was changed every 20 trials until 500 data were obtained for the scenario to obtain more accurate prediction values. The data were randomly divided into two disjoint subsets using 10-fold cross. The training set had 450 observations $(90 \%$ of data) and testing set had 50 (10\% of data). Consequently, the data of each deformation datum corresponding to each speed value were achieved to be used as teaching data for FITNET model.

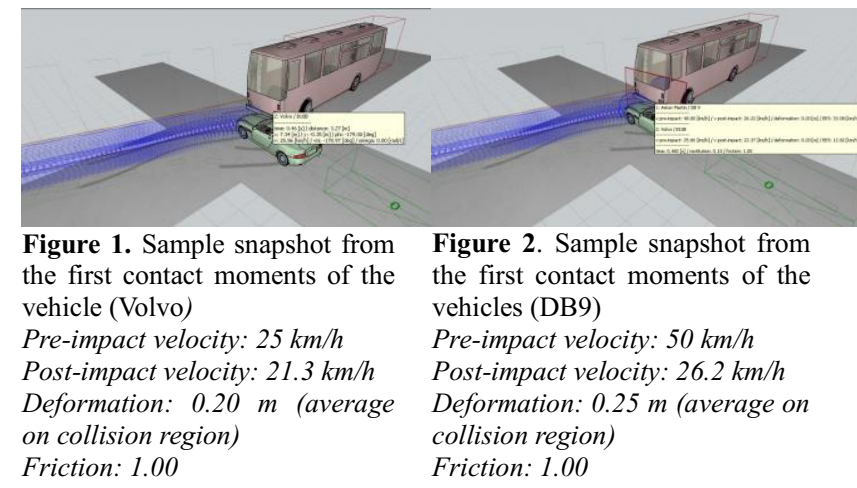

Two phases can be distinguished during the crash of a vehicle: there is a compression phase and a restitution phase. The compression phase lasts from the contact of the vehicle with an obstacle (another vehicle or anything else) to the point of maximum compression. During this phase, the energy is stocked until the maximum deformation. The restitution phase begins when deformation is maximum and ends when the vehicle separates from the obstacle. During this phase, the deformation energy is released [16]. 


\section{Overview of FITNET model}

The FITNET model consists of 3 layers (input, hidden and output). The input, hidden and output layers have 4 , 10 and 2 neurons respectively (Fig. 4). The tan-sigmoid activation function is used in FITNET model in the hidden layers. A pure-linear activation function is used in the output layer and Levenberg-Marquardt (LM) algorithm is utilized for training the networks. FITNET is also a modified form of Multilayer Feed Forward (MFFNN) with the default tan-sigmoid transfer function (Fig. 3a) in the hidden layer and linear transfer function (Fig. 3b) in the output layer.

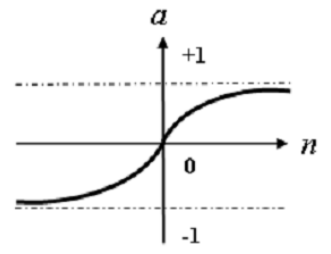

$a=$ Tansig $(n)=\frac{2}{1+e^{-2 n}}-1$

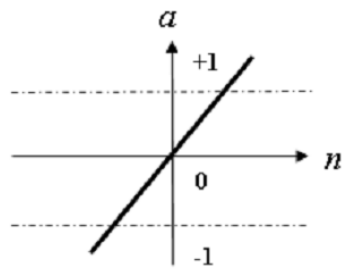

$a=$ Purelin $(n)$
Figure 3. Transfer functions for FITNET [17]

The other important parameters of the FITNET model are the number of epochs, the learning rate and momentum which were selected as 1000, 0.02 and 0.5, respectively.

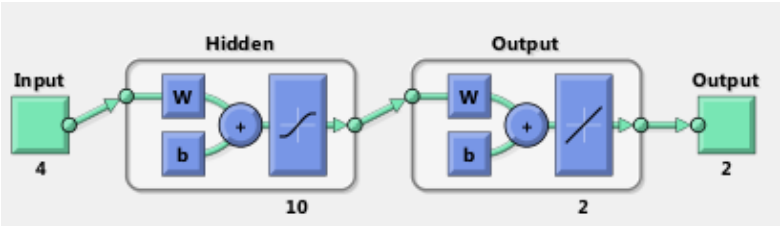

Figure 4. Architecture of FITNET model

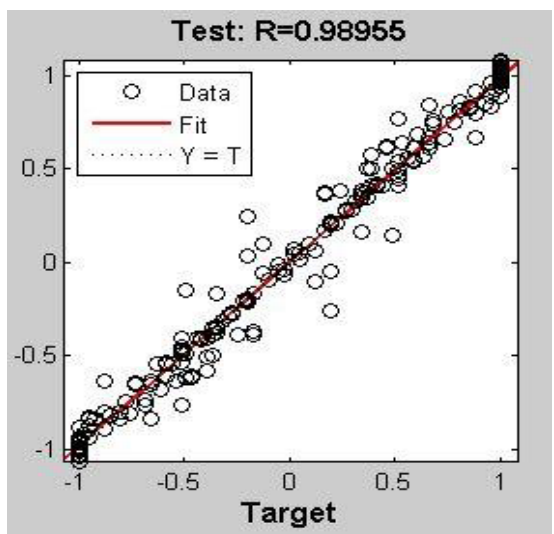

Figure 5. trainlm function, R values

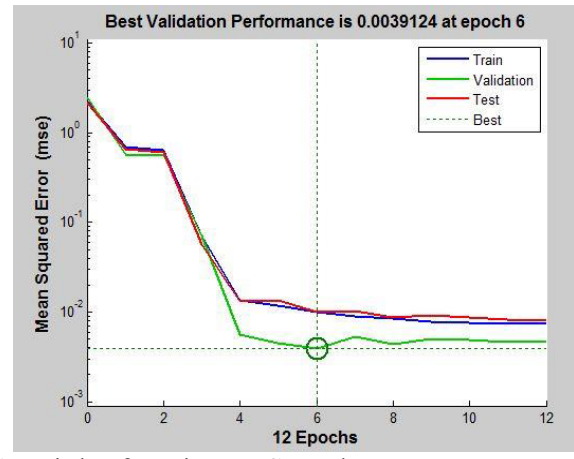

Figure 6. trainlm function, MSE values
Two different training algorithms (trainlm, trainrp) within FITNET were used in order to compare the $\mathrm{R}$ and MSE values. "trainlm" is a network training function that updates weight and bias values according to LevenbergMarquardt optimization. "trainlm" is often the fastest backpropagation algorithm in the toolbox, and is highly recommended as a first-choice supervised algorithm, although it does require more memory than other algorithms. "trainrp" is a network training function that updates weight and bias values according to the resilient backpropagation algorithm. Multilayer networks typically use sigmoid transfer functions in the hidden layers. These functions are often called "squashing" functions, because they compress an infinite input range into a finite output range. Sigmoid functions are characterized by the fact that their slopes must approach zero as the input gets large. This causes a problem when you use steepest descent to train a multilayer network with sigmoid functions, because the gradient can have a very small magnitude and, therefore, cause small changes in the weights and biases, even though the weights and biases are far from their optimal values. The purpose of the resilient backpropagation (Rprop) training algorithm is to eliminate these harmful effects of the magnitudes of the partial derivatives. Only the sign of the derivative can determine the direction of the weight update; the magnitude of the derivative has no effect on the weight update. The size of the weight change is determined by a separate update value. The update value for each weight and bias is increased by a factor "delt_inc" whenever the derivative of the performance function with respect to that weight has the same sign for two successive iterations [18]. It was observed that best validation performance and $\mathrm{R}$ values were achieved within trainlm training algorithm compared to trainrp function (Fig. 5, Fig. 6, Fig. 7 and Fig. 8).

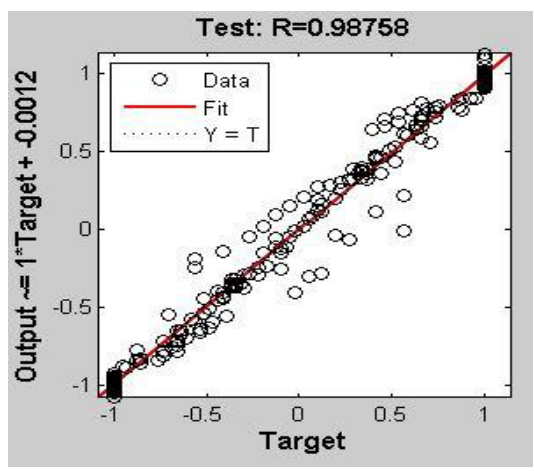

Figure 7. trainrp function, $R$ values

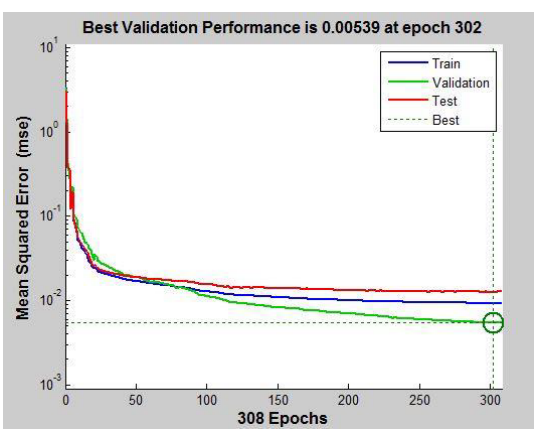

Figure 8. trainlm function, MSE values 
MSE in Equation 1 and $\mathrm{R}$ in Equation 2 were utilized in order to observe the prediction performance of the developed model by using MATLAB software [18].

$$
\begin{gathered}
M S E=\left[\sum_{1}^{N}\left(\frac{Q_{\mathrm{exp}}-Q_{\text {cal }}}{n}\right)^{2}\right] \\
R=\sqrt{\frac{\sum_{i=1}^{N}\left(Q_{\mathrm{exp}}-Q_{\text {cal }}\right)^{2}}{\sum_{i=1}^{N}\left(Q_{\mathrm{exp}}-\overline{Q_{\mathrm{exp}}}\right)^{2}}}
\end{gathered}
$$

where;

$Q_{\text {exp }}:$ observed value; $Q_{\text {cal }}:$ predicted value

$Q_{\text {exp }}$ : mean predicted value; $N$ : number of data points

\section{Results and discussion}

Deformation is the key parameter to estimate impact velocities. In simulation, 3 parameters related to crash analysis were also examined with deformation $(\varepsilon)$ which were deformation energy (E), pre-impact velocity $\left(v_{1}\right)$ and post-impact velocity $\left(v_{2}\right)$. Descriptive statistics for the dataset are given in Table 1.

Table 1. Descriptive statistics of the dataset

\begin{tabular}{|c|c|c|c|c|}
\hline \multirow{2}{*}{ Data } & \multicolumn{4}{|c|}{ Parameter } \\
\cline { 2 - 5 } & Mean & Max. & Min. & $\begin{array}{c}\text { Std. } \\
\text { Deviation }\end{array}$ \\
\hline $\mathrm{E}(\mathrm{J})$ & 63583.51 & 1013511.43 & 652.12 & 215873.74 \\
\hline $\boldsymbol{\varepsilon}(\mathbf{m})$ & $\mathbf{0 . 2 4}$ & $\mathbf{0 . 8 4}$ & $\mathbf{0 . 0 3}$ & $\mathbf{0 . 1 5}$ \\
\hline $\begin{array}{c}v_{1} \\
(\mathrm{~km} / \mathrm{h})\end{array}$ & 28.32 & 98.87 & 0 & 26.46 \\
\hline $\begin{array}{c}v_{2} \\
(\mathrm{~km} / \mathrm{h})\end{array}$ & 21.81 & 64.32 & 0.06 & 20.42 \\
\hline
\end{tabular}

However, for predicting the impact speeds using FITNET models, deformations formed on vehicles $(\varepsilon 1, \varepsilon 2)$ were used as input variables (training data) due to its bigger effect on speed analysis than the others and impact speeds (learning data) were the output variables. It was aimed that, in case of absence of clues about impact speed analysis such as skid marks, police reports, witnesses etc., deformation could be used as the main parameter for speed prediction. It may be possible that an application that can run on portable devices may be coded in order to estimate impact speeds by entering average deformations on the collision regions of vehicles.

Table 2. Descriptive statistics of the dataset

\begin{tabular}{|c|c|c|}
\hline Fold Number & MSE & R \\
\hline 1 & 0.409939 & 0.999843 \\
\hline 2 & 1.241129 & 0.999393 \\
\hline 3 & 0.223066 & 0.999924 \\
\hline 4 & 1.353711 & 0.999473 \\
\hline 5 & 1.451669 & 0.999366 \\
\hline 6 & 1.046015 & 0.999596 \\
\hline 7 & 1.359004 & 0.999425 \\
\hline
\end{tabular}

\begin{tabular}{|c|c|c|}
\hline Fold Number & MSE & R \\
\hline 8 & 1.528730 & 0.999434 \\
\hline 9 & 0.251186 & 0.999890 \\
\hline 10 & 0.935292 & 0.999574 \\
\hline Average & $\mathbf{0 . 9 8 9 5 5 4}$ & $\mathbf{0 . 9 9 9 5 9 2}$ \\
\hline
\end{tabular}

Within performance analysis of FITNET model, it was observed that average of MSE values was in acceptable range and average of $\mathrm{R}$ values was close to 1 (Table 2). The results showed that FITNET model with trainlm training function is an appropriate impact velocity predictor.

\section{Conclusions}

This study focuses on exhibiting new aspect and scientific approach for determining impact speeds of involvements in a sample most frequent accident occurring in Turkey without regarding to initiative and/or experience of experts. As subject matter, referring to direct proportion between speed and deformation energy, a new systematic approach for determining impact velocities was carried out. vCrash software was used to simulate the accident scene several times. The obtained dataset consisted of 4 parameters related to accident scene where deformations were used as main input parameter for FITNET model. The model was developed to predict impact speeds in which R and MSE values were also calculated. For the scenario, the FITNET model gave acceptable MSE and $\mathrm{R}$ values. Based on these approaches, various speed studies may be conducted within different prediction methods in a probable similar accident scene. It will be possible to analyze the impact speeds just by entering average deformation amounts into an application on a portable device at the accident scene without requirement of expensive reconstruction tools and it will be a guide for analyses of other accident types.

\section{Acknowledgment}

We would like to thank Cukurova University, Faculty of Engineering-Architecture, Automotive Engineering Laboratories and Scientific Research Unit for their resources that we were benefited from during conducting this study.

\section{References}

1. S. Miaou, H. Lum, Accident Anal. \& Prev., 25, 689709 (1993)

2. F. Temel, H. Ozcebe, Turkish Medic. Assoc., 15, 192-198 (2006)

3. E. Cangul, Development of an expert system for the quantification of fault rates in traffic accidents (Middle East Technical University, Engineering Faculty. MSc Thesis, 129 pages, 2010)

4. C. Murray, A. Lopez, K. Elissa. The Lancet, 349, 1498-1504 (1996)

5. Rep. of Turkey Ministry of Internal Affairs, Traffic Statistical Bulletin (2006) 
6. R. Wee, P. Rietveld, Transportation, 40, 295-314 (2013)

7. Y. Hayashi, H. Morisugi, Transport. Policy, 7, 73-88 (2000)

8. E. Karadayi, Uluslararası 1. Trafik ve Yol Güvenliği Kongresi, May, 8-12, Ankara, Turkey.

9. P. Koehn, A. Pauly, R. Fleck, M. Pischinger, T. Richter, M. Schnabel, R. Bartz, M. Wachinger, S. Schott, Automobiltechnische Zeitschrift Extra, 105, 96-105 (2003)

10. V.P. Kallberg. Motorcycle traffic accidents (Transport Res. Board, 49, 1986)

11. U. Buck, S. Naether, M. Braun, S. Bolliger, H. Friederich, C. Jackowski, E. Aghayev, P. Vock, R. Dirnhofer, M.J. Thali, Forensic Science Int., 170, 20-28 (2007)
12. G.A. Davis. Law, Prob. and Risk, 2, 69-89 (2003)

13. J. Xu, L. Yibing, L. Guangquan, W. Zhou, Accident Anal. \& Prev., 36, 783-788 (2009)

14. R.D. Raedt, I. Ponjaert-Kristoffersen, Accident Anal. \& Prev., 33, 809-819 (2001)

15. G. Yannis, J. Golias, E. Papadimitriou, Accident Anal. \& Prev., 37, 327-333 (2005)

16. E. Tomasch, Accident reconstruction guidelines (Graz University of Technology, 2004)

17. M.H. Beale, M.T. Hagan, H.B. Demuth, Neural network toolbox: for use with MatLab ( $7^{\text {th }}$ Ed., The MathWorks Inc. Michigan, USA, 2015)

18. MathWorks, MatLab. 2014. Available at: http://www.mathworks.com/products/matlab/. (Accessed May 4, 2015). 